\title{
A MARKOV TIME RELATED TO A PRIORITY SYSTEM
}

\author{
EDMOND J. VANDERPERRE
}

Received 4 February 2005; Revised 16 September 2005; Accepted 12 December 2005

We consider a basic renewable duplex system characterized by cold standby and subjected to a priority rule. Apart from a general stochastic analysis presented in the previous literature, we introduce a Markov time called the recovery time of the system. In order to obtain the corresponding Laplace-Stieltjes transform, we employ a stochastic process endowed with transition measures satisfying generalized coupled differential equations. The solution is provided by the theory of sectionally holomorphic functions.

Copyright (c) 2006 Edmond J. Vanderperre. This is an open access article distributed under the Creative Commons Attribution License, which permits unrestricted use, distribution, and reproduction in any medium, provided the original work is properly cited.

\section{Introduction}

Standby provides a powerful tool to enhance the reliability, availability, quality, and safety of operational plants, for example, $[4,8,16]$. However, in practice, standby systems are often subjected to a priority rule. For instance, the external power supply station of a technical plant has usually overall priority in operation with regard to an internal (local) power generator kept in cold or warm standby, for example, [4]. The local generator is only deployed if the external power station is down.

Cold or warm standby systems subjected to a priority rule and attended by a repair facility have received considerable attention in the previous literature, for example, $[2,3$, $5,6,9-15,17-19,21,23,24]$. We consider a basic duplex system composed of a priority unit (the $\mathbf{p}$-unit) and a nonpriority unit (the $\mathbf{n}$-unit) kept in cold standby until the $\mathbf{p}$-unit fails. The p-unit has overall (break-in) priority in operation with regard to the $\mathbf{n}$-unit, that is, the $\mathbf{n}$-unit is only deployed if the $\mathbf{p}$-unit is down. In order to avoid undesirable delays in repairing failed units, we suppose that the entire system (henceforth called the T-system) is attended by two different repairmen. Each repairman has his own particular task. Repairman $N$ is skilled in repairing the $\mathbf{n}$-unit, whereas repairman $P$ is an expert in repairing the $\mathbf{p}$-unit. Both repairmen are jointly busy if, and only if, both units are down. Otherwise, at least one repairman is idle. Figure 1.1 displays a functional block diagram of the T-system operating in standby.

Hindawi Publishing Corporation

Mathematical Problems in Engineering

Volume 2006, Article ID 92613, Pages 1-9

DOI 10.1155/MPE/2006/92613 


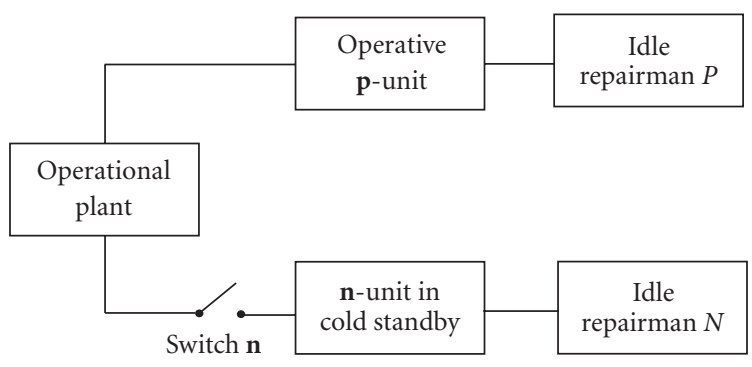

Figure 1.1. Functional block diagram of the T-system operating in standby.

Apart from a general stochastic analysis presented in the previous literature $[19,21$, 23], we introduce a Markov time called the recovery time of the T-system. The recovery time is the total (random) time needed to restore the $\mathbf{T}$-system from a prescribed risky state into the safe state (see the forthcoming formulation). In order to obtain the corresponding Laplace-Stieltjes transform, we employ a stochastic process endowed with transition measures satisfying generalized coupled partial differential equations. Our proposed transient equations are extending the steady-state equations presented by Vanderperre and Makhanov [23]. The explicit solution is provided by a refined application of the theory of sectionally holomorphic functions.

\section{Formulation}

Consider the basic T-system satisfying the following conditions. The $\mathbf{p}$-unit has a constant failure rate $\lambda>0$ and a general repair time distribution $R(\cdot), R(0)=0$. The corresponding failure-free time and repair time are denoted by $f$ and $r$. The operative $\mathbf{n}$ unit has a constant failure rate $\lambda_{s}>0$, but a zero failure rate in standby (the so-called cold standby) and a general repair time distribution $R_{s}(\cdot), R_{s}(0)=0$. The corresponding failure-free time and repair time are denoted by $f_{s}$ and $r_{s}$. The random variables $f, f_{s}, r, r_{s}$ are statistically independent. Any repair is perfect [7]. The switch-over time from standby to the operative state is instantaneous. Characteristic functions are formulated in terms of a complex transform variable. For instance,

$$
\mathbf{E} e^{i \omega r}=\int_{0}^{\infty} e^{i \omega x} d R(x), \quad \operatorname{Im} \omega \geq 0 .
$$

Note that

$$
\mathbf{E} e^{-i \omega r}=\int_{-\infty}^{0} e^{i \omega x} d\{1-R((-x)-)\}, \quad \operatorname{Im} \omega \leq 0
$$

The corresponding Fourier-Stieltjes transforms are called dual transforms. Without loss of generality (cf. [21, page 361]) we may assume that both $R$ and $R_{s}$ have bounded density functions (in the Radon-Nikodym sense) defined on $[0, \infty)$. 
In order to analyse the random behaviour of the $\mathbf{T}$-system, we employ a stochastic process $\left\{N_{t}, t \geq 0\right\}$ with arbitrary discrete state space $\{A, B, C, D\} \subset[0, \infty)$ characterized by the following events:

(i) $\left\{N_{t}=A\right\}$ : "the $\mathbf{p}$-unit is operative and the $\mathbf{n}$-unit is in cold standby at time $t$;"

(ii) $\left\{N_{t}=B\right\}$ : "the $\mathbf{n}$-unit is operative and the $\mathbf{p}$-unit is under repair at time $t$;"

(iii) $\left\{N_{t}=C\right\}$ : "the $\mathbf{p}$-unit is operative and the $\mathbf{n}$-unit is under repair at time $t$;"

(iv) $\left\{N_{t}=D\right\}$ : "both units are down at time $t$."

State $A$ is called the safe state. States $B$ and $C$ are called risky states and state $D$ is called the system down state. The non-Markovian process $\left\{N_{t}\right\}$ is defined on a filtered probability space $\{\Omega, \mathbf{B}, \mathbf{P}, F\}$ where the history $F:=\left\{F_{t}, t \geq 0\right\}$ satisfies the Dellacherie conditions:

(i) $F_{0}$ contains the $\mathbf{P}$-null sets of $\mathbf{B}$;

(ii) for all $t \geq 0, F_{t}=\bigcap_{u>t} F_{u}$, that is, the family $F$ is right continuous.

Consider the F-Markov time

$$
\theta:=\inf \left\{t>0: N_{t}=A \mid N_{0}=B, Z_{0}=0\right\}
$$

where $Z_{t}$ denotes the past repair time of the failed $\mathbf{p}$-unit being under progressive repair at time $t$. Note that we take the instant of the first failure as time origin, that is, $N_{0}=B, Z_{0}=$ $0, \mathbf{P}$-a.s. Thus, from $t=0$ onwards, $\theta$ is the total amount of time needed to restore the T-system from the risky state $B$ into the safe state $A$. $\theta$ is called the recovery time of the $\mathrm{T}$-system. In addition, note that our priority rule implies that a transition from the safe state $A$ into the risky state $C$ is only possible via state $D$.

A (vector) Markov characterization of the process $\left\{N_{t}, t \geq 0\right\}$ is piecewise and conditionally defined by

(i) $\left\{N_{t}\right\}$, if $N_{t}=A$ (i.e., if the event $\left\{N_{t}=A\right\}$ occurs);

(ii) $\left\{\left(N_{t}, X_{t}\right)\right\}$, if $N_{t}=B$, where $X_{t}$ denotes the remaining repair time of the $\mathbf{p}$-unit being under progressive repair at time $t$;

(iii) $\left\{\left(N_{t}, Y_{t}\right)\right\}$, if $N_{t}=C$, where $Y_{t}$ denotes the remaining repair time of the $\mathbf{n}$-unit being under progressive repair at time $t$;

(iv) $\left\{\left(N_{t}, X_{t}, Y_{t}\right)\right\}$, if $N_{t}=D$.

The state space of the underlying Markov process with absorbing state $A$ is given by

$$
\{A\} \bigcup\{(B, x) ; x \geq 0\} \bigcup\{(C, y) ; y \geq 0\} \bigcup\{(D, x, y) ; x \geq 0, y \geq 0\} .
$$

Let

$$
p_{A}(t):=\mathbf{P}\left\{N_{t}=A\right\}, \quad t \geq 0 .
$$

Finally, we introduce the measures:

$$
\begin{gathered}
p_{B}(t, x) d x:=\mathbf{P}\left\{N_{t}=B, X_{t} \in d x\right\}, \\
p_{C}(t, y) d y:=\mathbf{P}\left\{N_{t}=C, Y_{t} \in d y\right\}, \\
p_{D}(t, x, y) d x, d y:=\mathbf{P}\left\{N_{t}=D, X_{t} \in d x, Y_{t} \in d y\right\} .
\end{gathered}
$$


4 A Markov time related to a priority system

Note that, for instance,

$$
p_{D}(t)=\int_{0}^{\infty} \int_{0}^{\infty} d_{x} d_{y} \mathbf{P}\left\{N_{t}=D, X_{t} \leq x, Y_{t} \leq y\right\}=\int_{0}^{\infty} \int_{0}^{\infty} p_{D}(t, x, y) d x d y
$$

Notations 2.1. The real line and the complex plane are denoted by $\mathbb{R}$ and $\mathbb{C}$, with obvious superscript notations such as $\mathbb{C}^{+}, \mathbb{C}^{-}$. For instance, $\mathbb{C}^{+}:=\{\omega \in \mathbb{C}: \operatorname{Im} \omega>0\}$.

The indicator (function) of an event $\varepsilon \in \mathbf{B}$ is denoted by $\mathbf{1} \varepsilon$. The Heaviside unit-step function with the unit jump at $t=t_{0}>0$ is denoted by $U_{t_{0}}(t), t \geq 0$. Finally, let $[t]$ be the greatest integer function.

\section{Differential equations}

Applying Hokstad's supplementary variable technique in some time interval $[t, t+\Delta]$, $\Delta \downarrow 0$, for example, Alfa and Srinivasa [1] and taking the absorbing state $A$ into account, yields

$$
\begin{gathered}
p_{A}(t+\Delta)=p_{A}(t)+p_{B}(t, 0) \Delta+p_{C}(t, 0) \Delta+o(\Delta), \\
p_{B}(t+\Delta, x-\Delta)=p_{B}(t, x)\left(1-\lambda_{s} \Delta\right)+p_{D}(t, x, 0) \Delta+o(\Delta), \\
p_{C}(t+\Delta, y-\Delta)=p_{C}(t, y)(1-\lambda \Delta)+p_{D}(t, 0, y) \Delta+o(\Delta), \\
p_{D}(t+\Delta, x-\Delta, y-\Delta)=p_{D}(t, x, y)+\lambda_{s} p_{B}(t, x) \frac{d}{d y} R_{s}(y) \Delta+\lambda p_{C}(t, y) \frac{d}{d x} R(x) \Delta+o(\Delta) .
\end{gathered}
$$

Invoking the definition of directional derivative entails that for $t>0, x>0, y>0$,

$$
\begin{gathered}
\frac{d}{d t} p_{A}(t)=\rho_{B}(t, 0)+p_{C}(t, 0) \\
\left(\lambda_{s}+\frac{\partial}{\partial t}-\frac{\partial}{\partial x}\right) p_{B}(t, x)=p_{D}(t, x, 0) \\
\left(\lambda+\frac{\partial}{\partial t}-\frac{\partial}{\partial y}\right) p_{C}(t, y)=p_{D}(t, 0, y) \\
\left(\frac{\partial}{\partial t}-\frac{\partial}{\partial x}-\frac{\partial}{\partial y}\right) p_{D}(t, x, y)=\lambda_{s} p_{B}(t, x) \frac{d}{d y} R_{s}(y)+\lambda p_{C}(t, y) \frac{d}{d x} R(x) .
\end{gathered}
$$

Note that the initial condition $N_{0}=B, Z_{0}=0$ implies that $p_{B}(0, x)=d / d x R(x), x>0$. Moreover, $\mathbf{P}\{\theta \leq t\}=p_{A}(t)$.

Hence,

$$
\mathrm{E} e^{-z \theta}=\int_{0}^{\infty} e^{-z t} d p_{A}(t), \quad z \geq 0 .
$$




\section{Solution procedure}

It should be noted that our differential equations are well adapted to a Laplace-Fourier transformation. In fact, the $p$-functions are locally integrable with respect to $t$ and bounded on $[0, \infty)$. Consequently, the derivatives with respect to $t$ are also locally integrable.

Moreover, the integrability of the $p$-functions and the repair time densities with respect to $x, y$ on $[0, \infty)$ implies that the corresponding partial derivatives are also integrable on $[0, \infty)$. A Laplace-Fourier transform technique applied to the equations, taking the initial condition into account, reveals that for $\operatorname{Im} \omega \geq 0, \operatorname{Im} \eta \geq 0, z>0$,

$$
\begin{gathered}
\mathbf{E} e^{-z \theta}=\int_{0}^{\infty} e^{-z t} p_{B}(t, 0) d t+\int_{0}^{\infty} e^{-z t} p_{C}(t, 0) d t \\
\left(\lambda_{s}+z+i \omega\right) \int_{0}^{\infty} e^{-z t} \mathbf{E}\left(e^{i \omega X_{t}} \mathbf{1}\left\{N_{t}=B\right\}\right) d t+\int_{0}^{\infty} e^{-z t} p_{B}(t, 0) d t \\
=\mathbf{E} e^{i \omega r}+\int_{0}^{\infty} \int_{0}^{\infty} e^{-z t} e^{i \omega x} p_{D}(t, x, 0) d x d t \\
(\lambda+z+i \eta) \int_{0}^{\infty} e^{-z t} \mathbf{E}\left(e^{i \eta Y_{t}} \mathbf{1}\left\{N_{t}=C\right\}\right) d t+\int_{0}^{\infty} e^{-z t} p_{C}(t, 0) d t \\
=\int_{0}^{\infty} \int_{0}^{\infty} e^{-z t} e^{i \eta y} p_{D}(t, 0, y) d y d t \\
(z+i \omega+i \eta) \int_{0}^{\infty} e^{-z t} \mathbf{E}\left(e^{i \omega X_{t}} e^{i \eta Y_{t}} \mathbf{1}\left\{N_{t}=D\right\}\right) d t \\
+\int_{0}^{\infty} \int_{0}^{\infty} e^{-z t} e^{i \omega x} p_{D}(t, x, 0) d x d t+\int_{0}^{\infty} \int_{0}^{\infty} e^{-z t} e^{i \eta y} p_{D}(t, 0, y) d y d t \\
=\lambda \mathbf{E} e^{i \omega r} \int_{0}^{\infty} e^{-z t} \mathbf{E}\left(e^{i \eta Y_{t}} \mathbf{1}\left\{N_{t}=C\right\}\right) d t+\lambda_{s} \mathbf{E} e^{i \eta r_{s}} \int_{0}^{\infty} e^{-z t} \mathbf{E}\left(e^{i \omega X_{t}} \mathbf{1}\left\{N_{t}=B\right\}\right) d t .
\end{gathered}
$$

Adding (4.1) yields the functional equation:

$$
\begin{aligned}
(z+i \omega & +i \eta) \int_{0}^{\infty} e^{-z t} \mathbf{E}\left(e^{i \omega X_{t}} e^{i \eta Y_{t}} \mathbf{1}\left\{N_{t}=D\right\}\right) d t \\
& +\left(z+\lambda\left(1-\mathbf{E} e^{i \omega r}\right)+i \eta\right) \int_{0}^{\infty} e^{-z t} \mathbf{E}\left(e^{i \eta, Y_{t}} \mathbf{1}\left\{N_{t}=C\right\}\right) d t \\
& +\left(z+\lambda_{s}\left(1-\mathbf{E} e^{-i \eta r_{s}}\right)+i \omega\right) \int_{0}^{\infty} e^{-z t} \mathbf{E}\left(e^{i \omega X_{t}} \mathbf{1}\left\{N_{t}=B\right\}\right) d t \\
= & \mathbf{E} e^{i \omega r}-\mathbf{E} e^{-z \theta}, \quad \operatorname{Im} \omega \geq 0, \operatorname{Im} \eta \geq 0, z>0 .
\end{aligned}
$$

Inserting $\omega=-\tau+i z, \eta=\tau, \tau \in \mathbb{R}$ into the functional equation entails that

$$
\psi^{+}(\tau, z)-\psi^{-}(\tau, z)=\varphi(\tau, z),
$$


6 A Markov time related to a priority system

where

$$
\begin{gathered}
\psi^{+}(\tau, z):=\frac{1}{\gamma^{+}(\tau)} \int_{0}^{\infty} e^{-z t} \mathbf{E}\left(e^{i \tau Y_{t}} \mathbf{1}\left\{N_{t}=C\right\}\right) d t \\
\psi^{-}(\tau, z):=\frac{\tau}{\tau-i z} \frac{1}{\gamma^{-}(\tau, z)} \int_{0}^{\infty} e^{-z t} \mathbf{E}\left(e^{-(i \tau+z) X_{t}} \mathbf{1}\left\{N_{t}=B\right\}\right) d t \\
\varphi(\tau, z):=\frac{\mathbf{E} e^{-(i \tau+z) r}-\mathbf{E} e^{-z \theta}}{\gamma^{+}(\tau) \gamma^{-}(\tau, z)} \frac{1}{i \tau+z}, \\
\gamma^{+}(\tau):=1+\lambda_{s} \frac{\mathbf{E} e^{i \tau r_{s}}-1}{i \tau}, \quad \gamma^{+}(0):=1+\lambda_{s} \mathbf{E} r_{s}, \\
\gamma^{-}(\tau, z):=1+\lambda \frac{1-\mathbf{E} e^{-(i \tau+z) r}}{i \tau+z}, \quad z>0 .
\end{gathered}
$$

Remarks 4.1. Equation (4.3) constitutes a $z$-dependent Hilbert problem on the real line. Note that $z$-independent Hilbert problems, related to reliability engineering, have been solved by the theory of sectionally holomorphic functions. See [20,21] for further details. A similar approach shows that the $z$-dependent function

$$
\frac{1}{2 \pi i} \int_{\Gamma} \varphi(\tau, z) \frac{d \tau}{\tau-\omega}, \quad \omega \in \mathbb{C}, z>0
$$

is sectionally holomorphic in $\mathbb{C}$, provided that the singular Cauchy integral

$$
\frac{1}{2 \pi i} \int_{\Gamma} \varphi(\tau, z) \frac{d \tau}{\tau-u}, \quad u \in \mathbb{R}
$$

is defined as a Cauchy principal value in a double sense, see [21, the Appendix]. Finally, note that our statement holds for general repair time distributions! (cf. [21, Remarks, page 361]).

\section{The tail distribution}

In order to obtain $\mathbf{E} e^{-z \theta}$, we first remark that $\psi^{+}(\omega, z)$ is analytic in $\mathbb{C}^{+}$, boundedly continuous on $\mathbb{C}^{+} \cup \mathbb{R}$ and that

$$
\lim _{\substack{|\omega| \rightarrow \infty \\ 0 \leq \arg \omega \leq \pi}} \psi^{+}(\omega, z)=0 .
$$

Hence, by the Cauchy's theorem,

$$
\frac{1}{2 \pi i} \int_{\Gamma} \psi^{+}(\tau, z) \frac{d \tau}{\tau-\omega}=0, \quad \omega \in \mathbb{C}^{-}
$$

On the other hand, $\psi^{-}(\omega, z)$ is analytic in $\mathbb{C}^{-}$, boundedly continuous on $\mathbb{C}^{-} \cup \mathbb{R}$ and

$$
\lim _{\substack{|\omega| \rightarrow \infty \\ \pi \leq \arg \omega \leq 2 \pi}} \psi^{-}(\omega, z)=0 .
$$


Hence,

$$
\psi^{-}(\omega, z)=-\frac{1}{2 \pi i} \int_{\Gamma} \psi^{-}(\tau, z) \frac{d \tau}{\tau-\omega}, \quad \omega \in \mathbb{C}^{-}
$$

By (4.3), (5.2), (5.4), we obtain

$$
\psi^{-}(\omega, z)=\frac{1}{2 \pi i} \int_{\Gamma} \varphi(\tau, z) \frac{d \tau}{\tau-\omega}, \quad \omega \in \mathbb{C}^{-} .
$$

However, note that

$$
\lim _{\substack{\omega \rightarrow 0 \\ \omega \in \mathbb{C}^{-}, z>0}} \psi^{-}(\omega, z)=0 .
$$

So that by (4.4)

$$
\mathbf{E} e^{-z \theta}=\lim _{\substack{\omega \rightarrow 0 \\ \omega \in \mathbb{C}^{-}}} \frac{N^{-}(\omega, z)}{D^{-}(\omega, z)},
$$

where

$$
\begin{aligned}
& N^{-}(\omega, z):=\frac{1}{2 \pi i} \int_{\Gamma} \frac{\mathrm{E} e^{-(i \tau+z) r}}{(i \tau+z) \gamma^{+}(\tau) \gamma^{-}(\tau, z)} \frac{d \tau}{\tau-\omega}, \\
& D^{-}(\omega, z):=\frac{1}{2 \pi i} \int_{\Gamma} \frac{1}{(i \tau+z) \gamma^{+}(\tau) \gamma^{-}(\tau, z)} \frac{d \tau}{\tau-\omega} .
\end{aligned}
$$

Consequently, $\mathbf{E} e^{-z \theta}$ is completely determined.

Example 5.1. As an example, let $R(t)=1-e^{-\rho t}, \rho>0$, and $R_{s}(t)=U_{t_{0}}(t)$. Clearly, E $e^{-i \omega r}=$ $\rho /(\rho+i \omega), \omega \neq i \rho$, whereas $\mathbf{E} e^{i \omega r_{s}}=e^{i \omega t_{0}}$. Without loss of generality, we may take $t_{0}$ as time unit. A straightforward application of the residue theorem entails that

$$
\frac{1-\mathbf{E} e^{-z \theta}}{z}=\frac{\alpha(z)}{\beta(z)}
$$

where

$$
\begin{gathered}
\alpha(z):=1+\lambda_{s} \frac{1-e^{-z}}{z}, \quad \alpha(0):=1+\lambda_{s}, \\
\beta(z):=z+\rho+\lambda_{s}\left(1-a e^{-z}\right), \quad a:=\frac{\lambda+\rho e^{-(\lambda+\rho)}}{\lambda+\rho} .
\end{gathered}
$$

Hence,

$$
\mathbf{E} \theta=\frac{1+\lambda_{s}}{\rho+\lambda_{s}(1-a)}
$$


8 A Markov time related to a priority system

Note that the tail distribution $\mathbf{P}\{\theta>t\}$ is uniquely determined by the Laplace transform:

$$
\frac{1-\mathbf{E} e^{-z \theta}}{z}=\int_{0}^{\infty} e^{-z t} \mathbf{P}\{\theta>t\} d t, \quad z>0 .
$$

Moreover, $\mathbf{P}\{\theta>t\}$ is Lebesgue absolutely continuous on $(0, \infty)$. Hence, by the inversion theorem

$$
\mathbf{P}\{\theta>t\}=\frac{1}{2 \pi i} \int_{C_{\delta}} e^{z t} \frac{\alpha(z)}{\beta(z)} d z, \quad t>0
$$

where

$$
\int_{C_{\delta}} \cdots d z:=\lim _{T \rightarrow \infty} \int_{-i T+\delta}^{i T+\delta} \cdots d z, \quad \delta>0 .
$$

A straightforward evaluation of the Cauchy integral, similar to the methodology introduced by Vanderperre [22], reveals that

$$
\begin{aligned}
\mathbf{P}\{\theta>t\}= & \sum_{k=0}^{[t]} a^{k} e^{-\left(\rho+\lambda_{s}\right)(t-k)} \frac{\left(\lambda_{s}(t-k)\right)^{k}}{k !} \\
& +\sum_{k=0}^{[t]} a^{k}\left(\frac{\lambda_{s}}{\rho+\lambda_{s}}\right)^{k+1}\left\{1-e^{-\left(\rho+\lambda_{s}\right)(t-k)} \sum_{n=0}^{k} \frac{\left(\left(\rho+\lambda_{s}\right)(t-k)\right)^{n}}{n !}\right\} \\
& -\sum_{k=0}^{[t]-1} a^{k}\left(\frac{\lambda_{s}}{\rho+\lambda_{s}}\right)^{k+1}\left\{1-e^{-\left(\rho+\lambda_{s}\right)(t-k-1)} \sum_{n=0}^{k} \frac{\left(\left(\rho+\lambda_{s}\right)(t-k-1)\right)^{n}}{n !}\right\} .
\end{aligned}
$$

\section{Conclusion}

Our proposed priority system, subjected to general (bivariate) repair, can be analysed by elegant methods provided by the theory of sectionally holomorphic functions. However, the duplex system, subjected to general failure and repair time distributions, invokes an open (harsh) mathematical problem in the theory of statistical reliability engineering. The analysis of priority systems, subjected to arbitrary distributions, is far from complete.

\section{References}

[1] A. S. Alfa and T. S. S. Srinivasa Rao, Supplementary variable technique in stochastic models, Probability in the Engineering and Informational Sciences 14 (2000), no. 2, 203-218.

[2] J. R. Arora, Reliability of a two-unit priority standby redundant system with finite repair capability, IEEE Transactions on Reliability 25 (1976), 205-207.

[3] _ Reliability of several standby priority redundant systems, IEEE Transactions on Reliability 30 (1981), 123-132.

[4] A. Birolini, Quality and Reliability of Technical Systems, Springer, Berlin, 1994.

[5] J. A. Buzacott, Availability of priority redundant systems, IEEE Transactions on Reliability 20 (1971), 60-63.

[6] B. B. Fawzi and A. G. Hawkes, Availability of a series system with replacement and repair, Journal of Applied Probability 27 (1990), no. 4, 873-887. 
[7] I. B. Gertsbakh, Statistical Reliability Theory, Probability: Pure and Applied, vol. 4, Marcel Dekker, New York, 1989.

[8] B. Gnedenko and I. A. Ushakov, Probabilistic Reliability Engineering, John Wiley \& Sons, New York, 1995.

[9] L. R. Goel and S. K. Singh, Cost analysis of a two-unit priority standby system with imperfect switch, intermittent repair and arbitrary distributions, IEEE Transactions on Reliability 35 (1986), 585-589.

[10] R. Gupta, Analysis of a two-unit cold standby system with degradation and linearly increasing failure rates, International Journal of Systems Science 22 (1991), no. 11, 2329-2338.

[11] Y. Lam and Y. L. Zhang, Repairable consecutive-k-out-of- $n: F$ system with Markov dependence, Naval Research Logistics 47 (2000), no. 1, 18-39.

[12] W. Li and D. H. Shi, Reliability analysis of a two-unit parallel system with "preemptive priority" rule, Microelectronics and Reliability 33 (1993), no. 10, 1447-1453.

[13] H. Mine, Repair priority effect on the availability of a two-unit system, IEEE Transactions on Reliability 28 (1979), 325-326.

[14] T. Nakagawa and S. Osaki, Stochastic behaviour of a two-unit priority standby redundant system with repair, Microelectronics and Reliability 14 (1975), no. 3, 309-313.

[15] S. Osaki, Reliability analysis of a two-unit standby redundant system with priority, Canadian Journal of Operations Research 8 (1970), 60-62.

[16] H. Pham, Recent Advances in Reliability and Quality Engineering, World Scientific, Singapore, 2001.

[17] D. H. Shi and L. Liu, Availability analysis of a two-unit series system with a priority shut-off rule, Naval Research Logistics 43 (1996), no. 7, 1009-1024.

[18] Kh. Val'des Kastro, Optimal policies in a priority standby model, Moskow University Mathematics Bulletin 41 (1986), no. 4, 10-15, 102.

[19] E. J. Vanderperre, Reliability analysis of a two-unit cold standby redundant system subject to a priority rule, Cahiers du Centre d'Études de Recherche Opérationnelle, Operations Research 31 (1989), no. 1-2, 159-166.

[20] _ A Sokhotski-Plemelj problem related to a robot-safety device system, Operations Research Letters 27 (2000), no. 2, 67-71.

[21]_L_L Long-run availability of a two-unit standby system subjected to a priority rule, Bulletin of the Belgian Mathematical Society. Simon Stevin 7 (2000), no. 3, 355-364.

[22] Reliability analysis of a renewable multiple cold standby system, Operations Research Letters 32 (2004), no. 3, 288-292.

[23] E. J. Vanderperre and S. S. Makhanov, Long-run availability of a priority system: a numerical approach, Mathematical Problems in Engineering 2005 (2005), no. 1, 75-85.

[24] R. C. M. Yam, M. J. Zuo, and Y. L. Zhang, A method for evaluation of reliability indices for repairable circular consecutive k-out-of- $n: F$ systems, Reliability Engineering \& System Safety 79 (2003), no. 1, 1-9.

Edmond J. Vanderperre: Department of Decision Sciences, University of South Africa,

P.O. Box 392, Pretoria 0003, South Africa

E-mail address: evanderperre@yahoo.com 


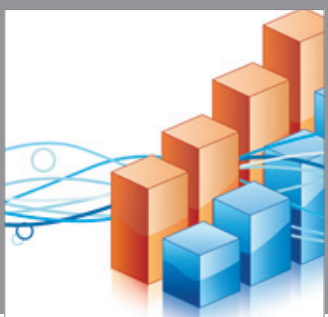

Advances in

Operations Research

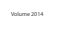

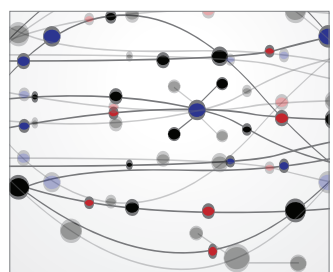

\section{The Scientific} World Journal
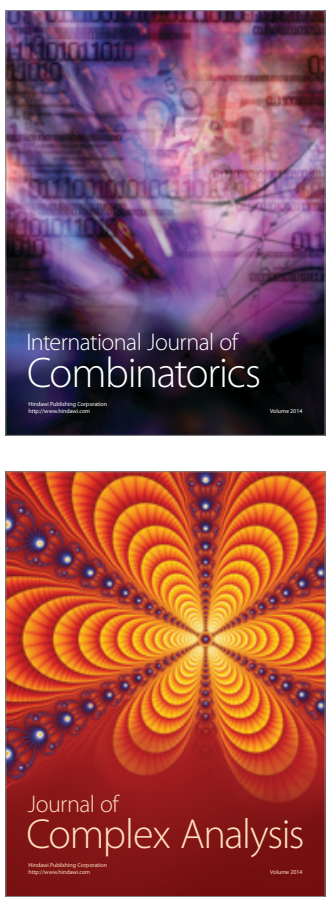

International Journal of

Mathematics and

Mathematical

Sciences
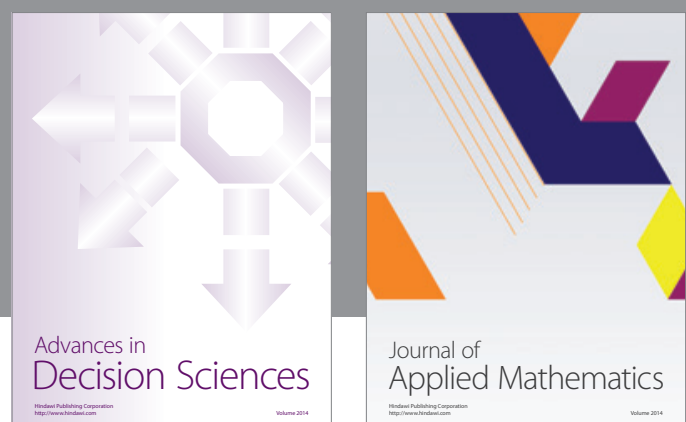

Journal of

Applied Mathematics
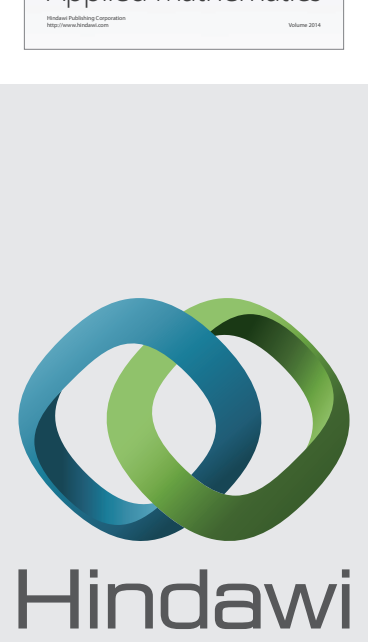

Submit your manuscripts at http://www.hindawi.com
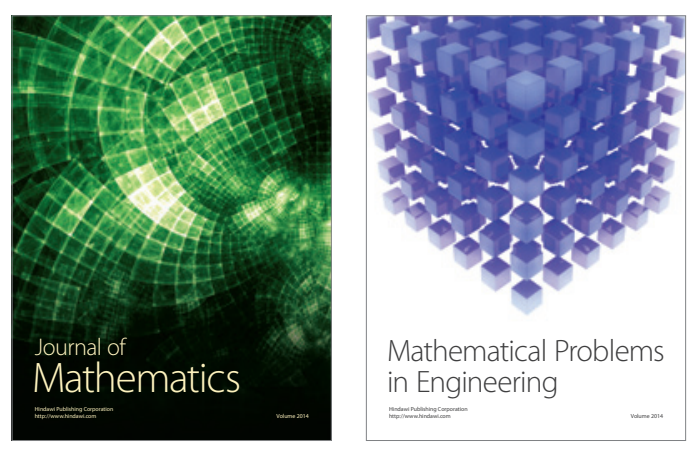

Mathematical Problems in Engineering
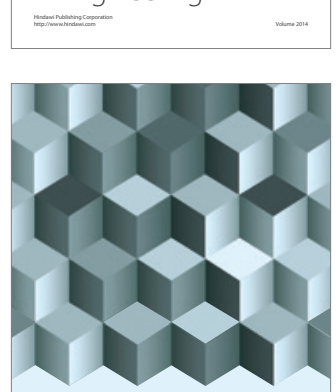

Journal of

Function Spaces
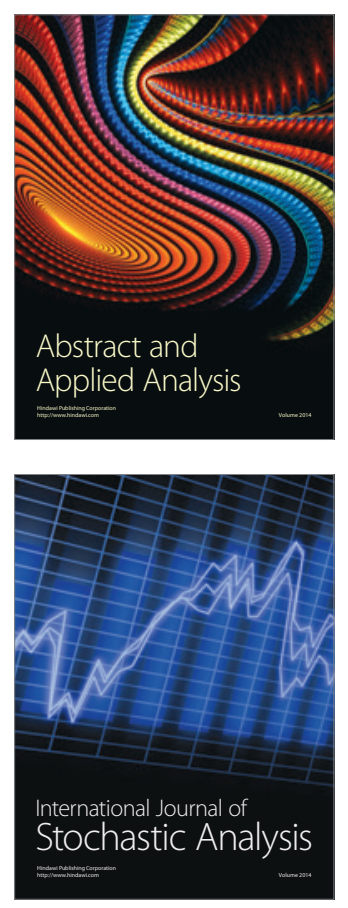

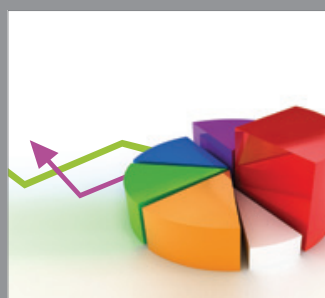

ournal of

Probability and Statistics

Promensencen
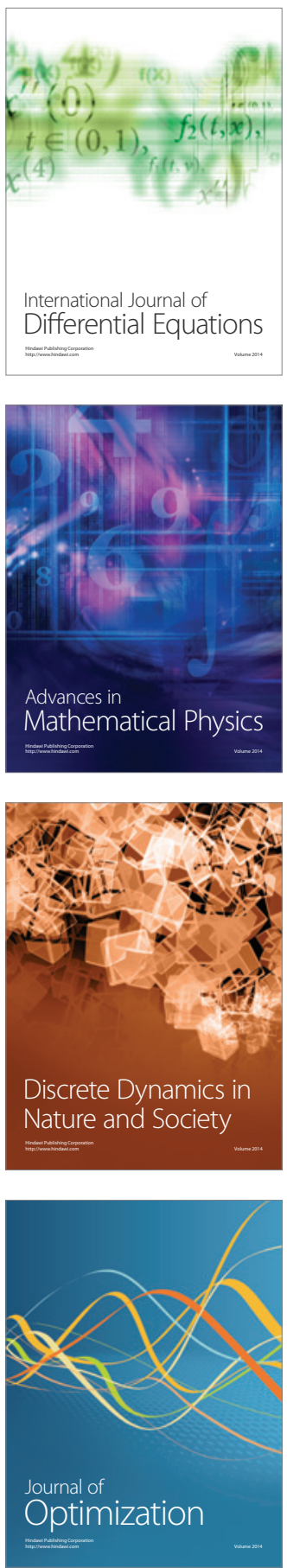